\title{
Simulation of Influences on the Dynamics of Transitional and Recurrent Processes of Complex Technical Systems
}

\author{
Bahram Ismailov Israfil
}

\begin{abstract}
The article presents the results of the analysis, simulation and evaluation of the vibration of complex coupled power equipment. Earlier, the sources, causes and nature of forced oscillations of a complex technical system with multidimensional chaotic behavior were considered. Ignoring the development of such processes can lead to overloading of power equipment and its parts and is fraught with unpredictable consequences. Using the examples of the influence and control of the dynamics of oscillating processes, various scenarios of the development of the vibration process are simulated.

In addition to traditional methods for assessing the development of controlled processes, additional methods and algorithms for visual and reflexive analysis have been proposed to help the decision maker in assessing the situation.

Index Terms: vibration, multi dimension alchaotic processes, Tsallis entropy, Poincare recurrence, stability, Lyapunov exponents
\end{abstract}

\section{INTRODUCTION}

The article is a continuation of the actual problem of controlling the vibration of a coupled complex power equipment [1], where the nature, description of the sources and conditions for the emergence of multidimensional chaotic vibration processes were cited. Based on the proven assertion about the sensitivity of a system with chaotic dynamics to small perturbations, we simulated impact algorithms having a different origin and a mathematical model is given. The impact algorithm, compiled according to the above mathematical model, is actually a corrective iterative algorithm that allows controlling the process of actions to obtain characteristics that meet the user's requirements. Taking into account some of the difficulties of parametric resolution of mathematical models, a method is proposed for studying the behavior of interacting systems by numerical methods. This approach allows you to combine together the conduct of analytical modeling and experimental studies that use the recorded observed activity of the controlled system.

\section{GENERAL CHARACTERISTICS OF INVESTIGATED NONLINEAR SYSTEMS}

The interest for controlling a complex technical system is caused by the need to maintain its operation in a safe mode. In a technical system, the constituent elements of which are interrelated and demonstrate chaotic dynamics, it is difficult to organize a steady and stable mode of operation.

\footnotetext{
Revised Manuscript Received on November 05, 2019.

Bahram Ismailov Israfil: Department of Instrumentation Engineering, Azerbaijan State Oil and Industry University, Baku, Azerbaijan Republic
}

The complex dynamics observed in such systems is difficult to control, it exposes the elements of the equipment to overloads, which ultimately leads to a reduction in the service life of the equipment and its rapid failure.

There are many numbers of analytical techniques are used to analyze, study and simulate the described nonlinear processes [2, 3].In the article, in addition to the well-known approaches, methods of analytical and numerical simulation of the control process are proposed using a thermodynamic-informational approach to assessing the characteristics of the development of multidimensional chaotic systems typical of the Open System [1, 4].

The simulated interaction processes are self-organizing, which are also non-equilibrium and take into account the processes of physical exchange between an Open System and a controlled environment and are considered from the standpoint of the exchange of information flows.

\section{METHODS AND MEANS FOR MODELING}

The mathematical model proposed in [1] is implemented in a sequential analysis and an iterative algorithm for finding modes that satisfy the requirements of the decision maker.

In studies on the proposed algorithm for controlling the behavior and development of the described chaotic systems, fragments of chaotic systems like Chen, Lee, Rabinovitch-Fabrikant, Levy, Liu, Chimera and other [5-9] were used as affecting systems. The specific features of these systems made it possible to realize the conceived scenarios for the development of dynamic processes.

To estimate the parameters of the signals, the computation of such informative parameters as the Lyapunov exponent, Tsallis entropy, stability, and fractal dimension were made.

In addition to the calculated values of the characteristics obtained, the visual results of the interactions performed in the form of recurrent diagrams are valuable. Nonlinear recurrent analysis of time series is one of the modern informative methods for visualizing the results of measurement experiments. Recurrent analysis is based on the fundamental property of dissipative dynamical systems recurrence, i.e. state recurrence. This property of processes in nature was noted by the French mathematician A. Poincare and was formulated in the form of the "recurrence theorem" [10]. The recurrent diagrams were proposed by the authors of [11] and allow you to map the phase trajectory of any dimension to a two-dimensional binary 
square matrix of $\mathrm{Nx} \mathrm{N}$, both coordinate axes of which are the axes of time. Another kind of recurrence diagram is a "distance plot", which displays the distances between the system states on the distance diagram as a cluster of points and a color palette. The distance chart carries information about the distances between the points of the trajectory. Other informative features of the recurrent diagrams are the topology and texture of the location of the points of the trajectory, the structure of which characterizes the hidden features of the process under study $[11,12,13]$.

The resulting images of recurrent diagrams allow you to visually assess the dynamics and trend of controlled processes and can also provide support in making decisions through the phenomenon of reflexive choice and visual thinking [14, 15].

A generalized analysis of informative parameters will allow a more reliable assessment of the influence of the vibration process, and in the case of its undesirable or dangerous development, make an informed decision on its correction.

\section{MODELING OF IMPACT AND CONTROL OF TRANSITION PROCESSES}

According to the proposed mathematical model [1]:

$s_{q}=\left[\left\{\sum_{i=1}^{N} s_{q}\left(D^{q} x\right)+\frac{1-\hat{q}}{n} \prod_{i=1}^{N} s_{q}\left(D^{q} x\right)\right\}+G M\right]$,

The algorithm for organizing the coherent behavior of a complex system is presented in Figure 1 and presents the stages of analysis and control and interactions for the study of transients.

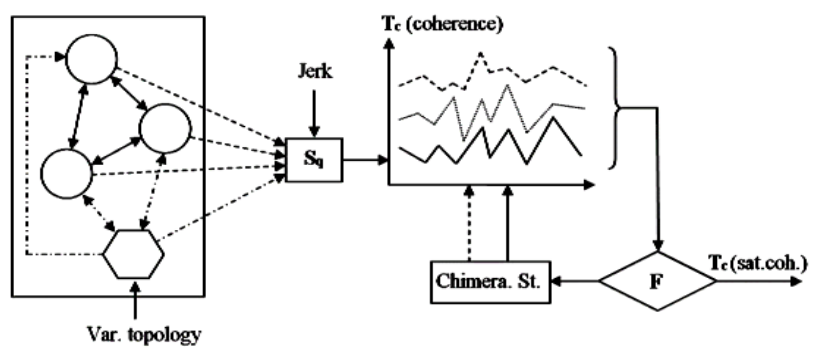

Figure 1. Organization of the coherence behavior systems.

To conduct research experiments on the impact on the base signal, we use the adaptive algorithm "measurement recognition - control", based on the features of nonlinear recurrent analysis (Figure 2).

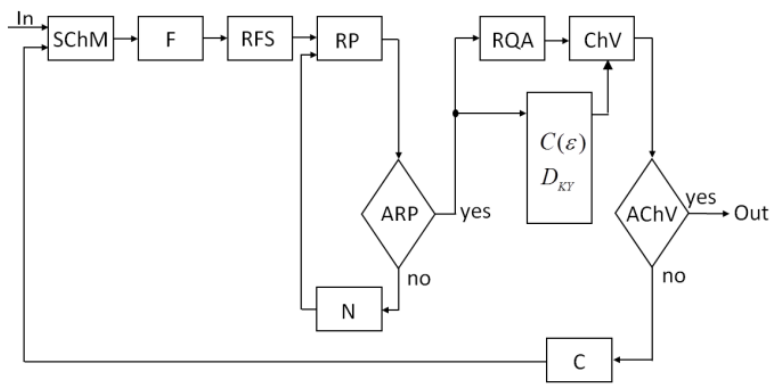

Figure 2. The structure of the adaptive system.
The structure of the system consists of:

- $\quad \mathrm{SChM}$ - stochastic and chaotic mappings;

- F- filter;

- $\quad$ RFS - phase space reconstruction;

- $\mathrm{RP}-$ recurrent diagramming;

- $\quad$ RQA - recurrent quantitative analysis;

- $\mathrm{ChV}$ - characteristic vector generation;

- $\quad \mathrm{ARP}-$ analysis of recurrent diagrams;

- $\mathrm{AChV}-$ analysis of characteristic vector;

- $\quad \mathrm{N}$ - norm setting $\left(L_{1}, L_{2}, L_{\infty}\right)$;

- $\mathrm{C}$ - calculation of: correlation integral $C(\varepsilon)$, new fractional dimension of Kaplan-Yorke $D_{K Y}$ and implementation filtering chaotic information, recurrent analysis of controlled processes.

Let's describe the composition of the interacting signals of the systems.

Figure 3 shows a fragment of pressure pulsation on the blades of the hydro-unit in the accelerating mode.

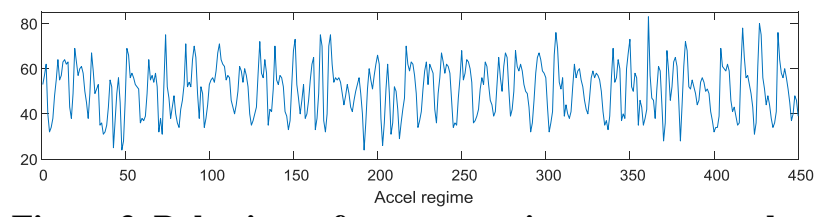

Figure 3. Pulsations of energy carrier pressure on the blades of the hydraulic unit guide vanes.

Energy carrier pressure pulsations on shovels of the guide vane of the hydro unit.

This mode is characterized as one of the most complex, as it is accompanied by a change in the vibration spectrum over time in a wide frequency range, which is caused by accelerating processes of rotation of the hydraulic unit before it reaches its operating speed.

Signals from the chaotic systems given below will be used for impacts on a basic signal:

The fractional-order chaotic Chen system as follows [5]:

$$
\begin{aligned}
& \frac{d^{\alpha} x}{d t^{\alpha}}=a(y-x)+w+N \\
& \frac{d^{\alpha} y}{d t^{\alpha}}=b x-x z+c y, \\
& \frac{d^{\alpha} z}{d t^{\alpha}}=x y-d z, \\
& \frac{d^{\alpha} w}{d t^{\alpha}}=y z+r w,
\end{aligned}
$$

where $a=35, b=7, c=12, d=3, r=0.5$ and $\alpha=0.9$.

Fractional-order hyperchaotic Liu system [6]: 


$$
\begin{aligned}
& \frac{d^{q} x}{d t^{q}}=100(y-x)+w, \\
& \frac{d^{q} y}{d t^{q}}=40 x-x z+0.5 w, \\
& \frac{d^{q} z}{d t^{q}}=-25 z+4 x^{2}-w, \\
& \frac{d^{q} w}{d t^{q}}=-d y-w,
\end{aligned}
$$

where $q=a . q ., 0<q<1$.

Levy processes used as models of strange kinetic phenomena in turbulent media [16].

Levy processes in terms of fractional dynamics reinforce the effect of the "transport-mixing-transport" fractional structure, evaluated by such parameters as the transport exponent, the Poincare return times, the dependence of the diffusion coefficient on the parameter in the standard display $[17,18,19]$.

A mathematical model of fractional Levy traffic can be represented as [20]:

$$
\widetilde{A}=m t+(\bar{\sigma} m)^{1 / 2} L_{\alpha, H(t)}
$$

where $m>0$ - is the average input rate constant; $\alpha \in(1,2)$ measurement of the thickness of the tails in the distribution; $\bar{\sigma}$ - scaling option.

Levy processes in terms of fractional dynamics enhance the effect of the "transport-mixing-transport" fractional structure, estimated by such parameters as the transport exponent, the Poincare return time, the dependence of the diffusion coefficient on the parameter in the standard display.

As one of the effect signals for resonant excitation, we use the state of the chimera. The state of the chimera was determined in [8, 9], it was defined as the coherent and incoherent oscillations. This condition is characterized as a manifestation of a complex transition process.

\section{THE RESULTS OF THE WORK OF AN ALGORITHM FOR SIMMULATION OF IMPACTS}

Figure 4 presents a comparative analysis of the effects.

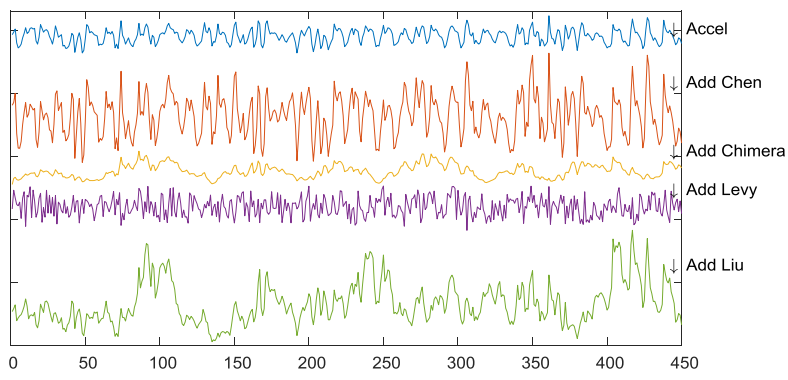

Figure 4. Results of effects on the vibration signal.

As can be seen from the results of the effects, the vibration signal was transformed, and its spectrum, entropy, fractal dimension, and stability changed accordingly.

Figure 5 shows as an example the results of calculations of informative parameters, such as: the entropy of Tsallis, the
Lyapunov exponent, the stability exponent and the value of the fractal dimension for the case of the effect of the Levy system signal on the vibration signal being monitored.

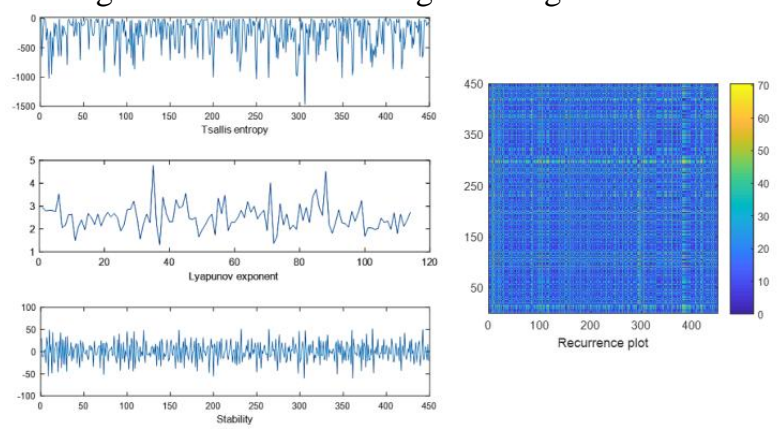

Figure 5. Tsallis entropy, Lyapunov exponent, Stability andRecurrenceplot reflecting the results of the impact

\section{of the Levy system signal, the value of the fractal dimension is equal to $\mathrm{D}=\mathbf{1 . 6 9 1 9}$.}

Of course, the choice of an appropriate impact remains with the decision maker and is associated with his experience and preferences in this area [13, 14].In practice, preference is given to the option that is most suitable for complex sustainability indicators (for example, a high-frequency resulting signal directed to a hydraulic unit with a large mass cannot have a significant change in its dynamics, while low-frequency effects may cause resonance responses, etc.). Alternatively, you can consider a mode in which a simpler dynamic is obtained. It is known that with the flow of signals with simple dynamics, the predictability of the behavior of the controlled system increases.

\section{CONCLUSION}

The use of analytical-numerical modeling and non-linear recurrent analysis allowed the analysis of vibration processes with the connection of the creative phenomenon of the reflexive choice of the decision-maker. It is known that the main advantage of the practical application of visual images of recurrent diagrams is their unusual information content. A visual assessment of the dynamics of the process being monitored, compared with the comprehension of a large volume of measurement information, allows you to quickly understand and evaluate the essence of the processes that are going on, to predict changes in their states. The proposed analysis, assessment and control of the vibration state of a complex technical system will allow more fully and more reliably reflect its real state, as well as reveal the hidden patterns of interconnections and interaction.

\section{REFERENCES}

1. Ismailov B.I. (2018). Analysis and Control of Dynamic Processes in Mechanical Parts of Power Equipment. International Journal of Mechanical and Production Engineering Research and Development (IJMPERD). Pp. 347-352.

2. Loskutov A.YU. (2001). Problemynelinejnojdinamiki II. Podavleniehaosaiupravleniedinamicheskimisistemami.

VestnikMoskovskogo Un-ta. Seriya 3. Fizika. Astronomiya, №3.- s. 3-21. 
3. Malineckij G.G., Potapov A.B (2002).Sovremennyeproblemynelinejnojdinamiki. M.: Editorial URSS. $360 \mathrm{~s}$.

4. Ismailov B.I. (2017). Thermodynamic - Informational Paradigm in the Context of the Formation of a Mathematical Model of Transient Processes in an Open System. European Journal of Engineering Research and Science Vol. 2, No. 10.Pp. 17-20.

5. Hegari A.S., Matouk A.E. (2011). Dynamical behaviors and synchronization in the fractional order hyperchaotic Chen system. Applied Mathematics Letters 24.Pp. 1938-1944.

6. Han Qiang at al. (2013). A four-dimension fractional order hyperchaotic system derived from Liu-system and its circuit research. Chinese Physics B. Volume 22, Number 2. Pp. 1-6.

7. Sprott J.C. (2003). Chaos and Time Series Analysis, Oxford University Press, Oxford. P. 507.

8. Panaggio Mark J. (2015). Chimera states: coexistence of coherence and incoherence in networks of coupled oscillators. IOP Publishing. Nonlinearity 28. R67-R87. doi:10.1088/0951-7715/28/3/R67.

9. Shanahan Murray. (2010). Metastable chimera states in community-structured oscillator networks. Chaos 20.Pp. 1-5.

10. Poincare A. (1912). Calcul des Probabilities. Paris: Gouthier-Villars.

11. Eckmann J. at al. (1987).Recurrence plots of dynamical systems //Europhys. Lett., 4. Pp. 973-977.

12. Bruce Hobbs and Alison Ord (2018). Nonlinear dynamical analysis of GNSS data: quantification, precursors and synchronization. Progress in Earth and Planetary Science. 35p.

13. Vladimirskij E.I., Ismajlov B.I (2011).Nelinejnyjrekurrentnyjanalizkakmatematicheskaya model' upravleniyahaoticheskimiprocessami. Informacionnyetekhnologii, №5, (177). s. 42-45.

14. Ismailov B.I. (2012). The Visual Control of Vibration Dynamic System. Eastern-European Journal of Enterprise Technologies. №5. Pp.25-30.

15. Vladimirskiy E.I. (2005). Otobrazhenieprintsipovrefleksii v matematicheskoymodeliprinyatiyaudovletvoritelnyihresheniy. Tr. IV Mezhd. Konferentsii «Identifikatsiyasistemizadachiupravleniya», SICPRO'05. Moskva 25-28 yanvarya. M.: Institut problem upravleniyaim. V.A.Trapeznikova RAN. Pp. 1681-1688

16. Zeleny L.M., Milovanov A.V (2004).Fraktalnayatopologiyaistrannayakinetika: otteoriiperkolyatsii k problemamkosmicheskoyelektrodinamiki. [Tekst]// UFN, t. 174, №8, s. 810-850.

17. Anishchenko V.S., Astakhov S.V. (2013) TeoriyavozvratovPuankareieyoprilozheniye zadachamnelineynoyfiziki. [Tekst] //UFN 183 1009-1028.

18. Vladimirsky E.I. (2012). Poincare Returns Times in the Interaction of Chaotic and Stochastic Systems. Eastern-European Journal of Enterprise Technologies. №6/4 (60). Pp.4-8.

19. Vladimirsky E., Ismailov B. (2014). Fractional Structure « MIXING TRANSPORT » as Open System. Eastern-European Journal of Enterprise Technologies. №4/4 (70). Pp.4-9.

20. Laskin N. at al. (2002). Fractional Levy Motion and Its Applications to Network Traffic Modeling. [Text].// Computer Networks, vol. 40, issue 3, pp. 363-375. Doi>10.1016/S1389-1286(02)00300-6. 